$\xi=$ 臬

\title{
A general solution to non-collinear equilibria in terms of largest root (к) of confocal oblate spheroid
}

\author{
M. Javed Idrisi * \\ Department of Mathematics, Al-Falah University, Faridabad (Haryana) -121004, India \\ *Corresponding author E-mail: mjavedidrisi@gmail.com
}

\begin{abstract}
This paper deals with the existence of non-collinear equilibria in restricted three-body problem when less massive primary is an oblate spheroid and the potential of oblate spheroid is in terms of largest root of confocal oblate spheroid. This is found that the non-collinear equilibria are the solution of the equations $r_{1}=n^{-2 / 3}$ and $\kappa=1-a^{2}$, where $r_{1}$ is the distance of the infinitesimal mass from more massive primary, $n$ is mean-motion of primaries, $a$ is semi axis of oblate spheroid and $\kappa$ is the largest root of the equation of confocal oblate spheroid passes through the infinitesimal mass.
\end{abstract}

Keywords: Celestial Mechanics; Restricted Three-Body Problem; Libration Points; Oblate Spheroid; Confocal Oblate Spheroid.

\section{Introduction}

The restricted problem of three-body describes the motion of infinitesimal mass moving in the gravitational field of two massive primaries in the same plane or out of plane called two dimensional or three dimensional problem accordingly. The primaries are revolving around their center of mass either in circular or elliptical orbits under the influence of their mutual gravitational attraction. If the orbit of the primaries around their center of mass is elliptic, problem is said to be elliptic restricted three-body problem (ER3BP or ERTBP) and if the orbit of the primaries around their center of mass is circular, problem is said to be circular restricted three-body problem or restricted three-body problem, denoted by CR3BP or CRTBP or RTBP or R3BP.

The problem possesses five equilibrium points out of which three are collinear and two non-collinear. The collinear libration points are unstable while non-collinear are stable for the mass ratio $\mu \leq$ 0.038520896505 [3].Some studies related to the equilibrium points in R3BP or ER3BP, taken into account the oblateness and triaxiality of the primaries, Coriolis and Centrifugal forces, variation of the masses of the primaries and the infinitesimal mass etc. are discussed by Danby [2]; Szebehely [3]; Vidyakin [4]; Sharma [5]; Subbarao and Sharma [6]; Sharma et.al. [7]; Choudhary R. K. [8]; Cid R. et. al. [10]; El-Shaboury [11]; Bhatnagar et al. [12]; Selaru D. et.al. [13]; Markellos et al. [14]; Subbarao and Sharma [15]; Khanna and Bhatnagar [16, 17]; Roberts G.E. [18]; Oberti and Vienne [19]; Sosnytskyi [20]; Perdiou et. al. [21]; Arredondo et.al. [22]; Idrisi and Taqvi [23]; Idrisi [24]; Idrisi and Amjad [25]. In 1958, W. D. MacMillan [1] gave a theory to find out the potential of ellipsoid in terms of largest root of confocal ellipsoid. Let $a$, $b, c$ be the semi-axes of the given oblate spheroid such that $a=b$ $>c,(x, y, z)$ the co-ordinates of the external point $P$ and $a^{\prime}, b^{\prime}, c^{\prime}$ $\left(a^{\prime}=b^{\prime}>c^{\prime}\right)$ be the semi-axes of the confocal oblate spheroid passes through $P$.

Let the equation of the oblate spheroid be

$$
\frac{\xi^{2}+\eta^{2}}{a^{2}}+\frac{\zeta^{2}}{c^{2}}=1
$$

where $\xi, \eta, \zeta$ are the principal axes of the oblate spheroid.

The equation of the confocal oblate spheroid whose semi-axes are $a^{\prime}, b^{\prime}$ and $c^{\prime}$ and passes through the point $P(x, y, z)$ is

$$
\frac{x^{2}+y^{2}}{a^{1^{2}}}+\frac{z^{2}}{c^{c^{2}}}=1
$$

Since the oblate spheroids are confocal, we have $a^{\prime 2}=a^{2}+\kappa$, $b^{-2}=b^{2}+\kappa$ and $c^{-2}=c^{2}+\kappa$. Therefore, the Equation (2) becomes

$$
\frac{x^{2}+y^{2}}{a^{2}+\kappa}+\frac{z^{2}}{c^{2}+\kappa}=1
$$

The potential of the oblate spheroid with axes $a$ and $c$ and density $\sigma$ at an external point $P(x, y, z)$ in terms of $\kappa$ is given by (MacMillan [1])

$$
\begin{aligned}
V= & \frac{2 \pi \sigma a^{2} c}{\sqrt{a^{2}-c^{2}}}\left(1-\frac{x^{2}+y^{2}-2 z^{2}}{2\left(a^{2}-c^{2}\right)}\right) \sin ^{-1} \sqrt{\frac{a^{2}-c^{2}}{a^{2}+\kappa}}+ \\
& \frac{\pi \sigma a^{2} c \sqrt{c^{2}+\kappa}}{a^{2}-c^{2}} \frac{x^{2}+y^{2}}{a^{2}+\kappa}-\frac{\pi \sigma a^{2} c}{a^{2}-c^{2}} \frac{2 z^{2}}{\sqrt{c^{2}+\kappa}},
\end{aligned}
$$

where $\kappa$ is defined in the Eqn. (3).

The aim of this paper is to find out non-collinear equilibria in restricted three-body problem when less massive primary is an oblate spheroid, using the potential in terms of $\kappa$ (Eqn. 4) and also verify the results with previous studies such as [6] and [9]. 


\section{Equations of motion}

Let $m_{2}$ be an oblate spheroid whose axes are $a, b$ and $c(a=b>c)$ and $m_{1}$ a point mass $\left(m_{1}>m_{2}\right)$, are moving in the circular orbits around their center of mass $O$. An infinitesimal mass $m_{3}$ is moving in the plane of motion of $m_{1}$ and $m_{2}$. The distances of $m_{3}$ from $m_{1}$, $m_{2}$ and $O$ are $\boldsymbol{r}_{\mathbf{1}}, \boldsymbol{r}_{\mathbf{2}}$ and $\boldsymbol{r}$ respectively. The principal axes of spheroid remains parallel to the synodic axes Oxyz throughout the motion and the equatorial plane of $m_{2}$ is coincide with the plane of motion of $m_{1}$ and $m_{2}$. Let the line joining $m_{1}$ and $m_{2}$ be taken as $X$ -axis and $O$ their center of mass as origin. Let the line passing through $O$ and perpendicular to $O X$ and lying in the plane of motion $m_{1}$ and $m_{2}$ be the $Y$-axis. Let us consider a synodic system of co-ordinates $O x y z$ initially coincide with the inertial system $O X Y Z$, rotating with angular velocity $\omega$ about $Z$-axis (the $z$-axis is coincide with $Z$-axis). We wish to find the equations of motion of $m_{3}$ using the terminology of Szebehely (1967) in the synodic co-ordinate system and dimensionless variables i.e. the distance between the primaries is unity, the unit of time $t$ is such that the gravitational constant $G=1$ and the sum of the masses of the primaries is unity i.e. $m_{1}+m_{2}=1$.

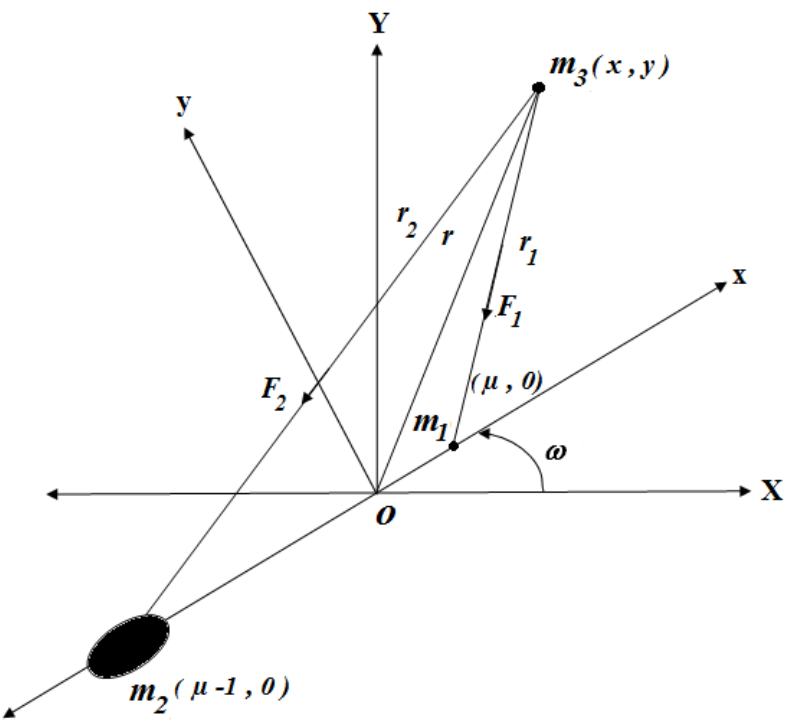

Fig. 1: The configuration of the R3BP when $m_{2}$ is an oblate spheroid

The potential of the oblate spheroid $m_{2}$ at $P(x, y)$ in our case is therefore given by,

$$
\begin{aligned}
-V= & \frac{2 \pi \sigma a^{2} c}{\sqrt{a^{2}-c^{2}}}\left(1-\frac{x^{2}+y^{2}}{2\left(a^{2}-c^{2}\right)}\right) \sin ^{-1} \sqrt{\frac{a^{2}-c^{2}}{a^{2}+\kappa}}+ \\
& \frac{\pi \sigma a^{2} c \sqrt{c^{2}+\kappa}}{a^{2}-c^{2}} \frac{x^{2}+y^{2}}{a^{2}+\kappa},
\end{aligned}
$$

where $\kappa$ is defined by the Eqn.

$$
\frac{(x+1-\mu)^{2}+y^{2}}{a^{2}+\kappa}+\frac{z^{2}}{c^{2}+\kappa}=1
$$

and

$$
\mu=\frac{m_{2}}{m_{1}+m_{2}} \leq \frac{1}{2}
$$

The equations of the motion of $m_{3}$ in the synodic co-ordinate system and dimensionless variables are: $m_{3}\left[\partial^{2} \boldsymbol{r} / \partial t^{2}+2 \boldsymbol{\omega} \times \partial \boldsymbol{r} / \partial t+\partial \boldsymbol{\omega} / \partial t \times \boldsymbol{r}+\boldsymbol{\omega} \times(\boldsymbol{\omega} \times \boldsymbol{r})\right]=\boldsymbol{F}=\boldsymbol{F}_{\mathbf{1}}+\boldsymbol{F}_{2}$

where

$\boldsymbol{r}=x \boldsymbol{i}+y \boldsymbol{j}$,

$\boldsymbol{\omega}=n \boldsymbol{k}$,

$\boldsymbol{F}=$ Total force acting on $m_{3}=\boldsymbol{F}_{\mathbf{1}}+\boldsymbol{F}_{\mathbf{2}}$

$\boldsymbol{F}_{1}=$ Gravitational force exerted on $m_{3}$ due to $m_{1}$,

$\boldsymbol{F}_{\mathbf{2}}=$ Gravitational force exerted on $m_{3}$ due to $m_{2}$

We first calculate the L.H.S. of the Equation (8) in the Cartesian form as follows:

$\partial \boldsymbol{r} / \partial t=\dot{x} \boldsymbol{i}+\dot{y} \boldsymbol{j}$,

Relative acceleration $=\partial^{2} \boldsymbol{r} / \partial t^{2}=\ddot{x} \boldsymbol{i}+\ddot{y} \boldsymbol{j}$,

$\boldsymbol{\omega} \times \boldsymbol{r}=-n y \boldsymbol{i}+n x \boldsymbol{j}$,

Coriolis acceleration $=\omega \times \partial \boldsymbol{r} / \partial t=-n \dot{y} \boldsymbol{i}+n \dot{x} \boldsymbol{j}$,

Centrifugal acceleration $=\omega \times(\boldsymbol{\omega} \times \boldsymbol{r})=-n^{2}(x \boldsymbol{i}+y \boldsymbol{j})$,

$\partial \omega / \partial t=0$

Euler's acceleration $=\partial \boldsymbol{\omega} / \partial t \times \boldsymbol{r}=\mathbf{0}$.

Thus, the L.H.S. of the Equation (7) becomes $m_{3}\left[\left(\ddot{x} \boldsymbol{i}+\ddot{y}_{j}\right)-2 n(\dot{y} \boldsymbol{i}-\dot{x} \boldsymbol{j})-n^{2}(x \boldsymbol{i}+y \boldsymbol{j})\right]$

Now, we calculate the R.H.S. of the Equation (8) as follows:

Let the gravitational potential of $m_{1}$ and $m_{2}$ at $m_{3}$ be $V_{1}$ and $V_{2}$ respectively, therefore

$$
\begin{aligned}
-V_{1}= & \frac{G m_{1} m_{3}}{r_{1}}, \\
-V_{2}= & \frac{3 G m_{2} m_{3}}{4}\left[\frac{2}{\sqrt{a^{2}-c^{2}}}\left(1-\frac{x^{2}+y^{2}}{2\left(a^{2}-c^{2}\right)}\right) \sin ^{-1} \sqrt{\frac{a^{2}-c^{2}}{a^{2}+\kappa}}+\right. \\
& \left.\frac{\sqrt{c^{2}+\kappa}}{a^{2}-c^{2}} \frac{x^{2}+y^{2}}{a^{2}+\kappa}\right],
\end{aligned}
$$

where

$m_{2}=\frac{4 \pi a^{2} c \sigma}{3}$,

Gravitational force exerted on $m_{3}$ due to $m_{1}$ and $m_{2}$ is given by $\boldsymbol{F}_{\mathbf{1}}=-\left(\partial V_{1} / \partial x \boldsymbol{i}+\partial V_{1} / \partial y \boldsymbol{j}\right)$

$\boldsymbol{F}_{\mathbf{2}}=-\left(\partial V_{2} / \partial x \boldsymbol{i}+\partial V_{2} / \partial y \boldsymbol{j}\right)$.

Thus, the Equation (7) becomes

$m_{3}\left[(\ddot{x} \boldsymbol{i}+\ddot{y} \boldsymbol{j})-2 n(\dot{y} \boldsymbol{i}-\dot{x} \boldsymbol{j})-n^{2}(x \boldsymbol{i}+y \boldsymbol{j})\right]=-\left(\partial V_{1} / \partial x+\right.$ $\left.\partial V_{2} / \partial x\right) \boldsymbol{i}-\left(\partial V_{1} / \partial y+\partial V_{2} / \partial y\right) \boldsymbol{j}$

i. $e$

$\ddot{x}-2 n \dot{y}=n^{2} x-\frac{1-\mu}{r_{1}^{3}}(x-\mu)-\frac{3 \mu(x+1-\mu)}{2\left(a^{2}-c^{2}\right)} \times$

$$
\left[\frac{1}{\sqrt{a^{2}-c^{2}}} \sin ^{-1} \sqrt{\frac{a^{2}-c^{2}}{a^{2}+\kappa}}-\frac{\sqrt{c^{2}+\kappa}}{\left(a^{2}+\kappa\right)}\right],
$$

and

$$
\ddot{y}+2 n \dot{x}=y\left[\begin{array}{l}
n^{2}-\frac{1-\mu}{r_{1}^{3}}-\frac{3 \mu}{2\left(a^{2}-c^{2}\right)} \times \\
{\left[\begin{array}{l}
\frac{1}{\sqrt{a^{2}-c^{2}}} \sin ^{-1} \sqrt{\frac{a^{2}-c^{2}}{a^{2}+\kappa}}-\frac{\sqrt{c^{2}+\kappa}}{\left(a^{2}+\kappa\right)}
\end{array}\right],}
\end{array}\right],
$$

where $n$ is the mean-motion of the primaries. 


$$
\begin{aligned}
& 1-\mu=\frac{m_{1}}{m_{1}+m_{2}}, \\
& r_{1}^{2}=(x-\mu)^{2}+y^{2}, \\
& r_{2}^{2}=(x+1-\mu)^{2}+y^{2} .
\end{aligned}
$$

Now, we define a function $\Omega$ such that

$\Omega=\frac{n^{2}}{2}\left(x^{2}+y^{2}\right)+\frac{1-\mu}{r_{1}}+V$

where $V$ is defined in the Eqn. (5).

Hence, the Eqns. (8) and (9) become

$\ddot{x}-2 n \dot{y}=\Omega_{x}$,

$\ddot{y}+2 n \dot{x}=\Omega_{y}$,

where $\Omega_{\mathrm{x}}$ and $\Omega_{\mathrm{y}}$ are the partial derivatives of $\Omega$ with respect to $x$ and $y$ respectively.

The integral analogous to Jacobi integral is

$\left(\dot{x}^{2}+\dot{y}^{2}\right)=2 \Omega-C$.

\section{Calculation of the mean-motion $n$ of the primaries}

Since the primaries $m_{1}$ and $m_{2}$ are moving in the circular orbits around their center of mass $\mathrm{O}$. Therefore, the mean-motion $\mathrm{n}$ of the primaries is given by,

$n^{2}=\frac{F}{m_{1} m_{2}}$

where $F$ is the gravitational force acting on $m_{1}$ due to $m_{2}$.

From Eqn. (6), the value of the largest root $\kappa$ at $m_{1}(\mu, 0,0)$ is $\kappa=1-a^{2}$

Thus, the mean-motion $n$ of the primaries is

$n^{2}=\frac{3}{2\left(a^{2}-c^{2}\right)}\left[\frac{1}{\sqrt{a^{2}-c^{2}}} \sin ^{-1} \sqrt{a^{2}-c^{2}}-\sqrt{1-\left(a^{2}-c^{2}\right)}\right]$

In terms of infinite series, mean-motion of the primaries is given by

$n^{2}=1+\frac{3\left(a^{2}-c^{2}\right)}{10}+\frac{9\left(a^{2}-c^{2}\right)^{2}}{56}+\frac{5\left(a^{2}-c^{2}\right)^{3}}{48}+\frac{105\left(a^{2}-c^{2}\right)^{4}}{1408}+\ldots$

Let $A=\left(a^{2}-c^{2}\right) / 5 \ll<1$ be the oblateness factor, therefore

$n^{2}=1+\frac{3}{2} A+\frac{45}{56} A^{2}+\frac{25}{48} A^{3}+\frac{525}{1408} A^{4}+\ldots$

On neglecting the second and higher powers in $A$, we have

$n^{2}=1+\frac{3 A}{2}$

The results are agreed with [6]. For $A=0$, the results are in conformity of the classical case of the restricted three-body problem [3]. From Fig. 2, as oblateness factor $A$ increases, the meanmotion of the primaries also increases.

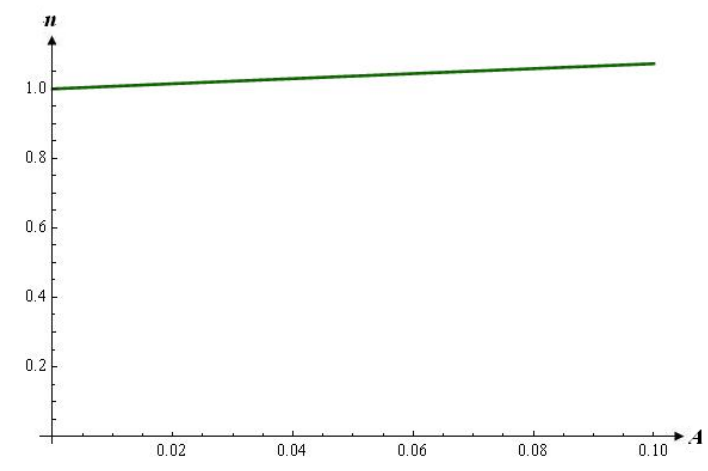

Fig. 2: $A$ versus $n$

\section{Non-collinear equilibrium solution}

The non-collinear equilibria are the solution of the equations $\Omega_{x}=$ 0 and $\Omega_{y}=0, y \neq 0$ i.e.

$n^{2} x-\frac{1-\mu}{r_{1}^{3}}(x-\mu)-\frac{3 \mu(x+1-\mu)}{2\left(a^{2}-c^{2}\right)} \times$

$\left[\frac{1}{\sqrt{a^{2}-c^{2}}} \sin ^{-1} \sqrt{\frac{a^{2}-c^{2}}{a^{2}+\kappa}}-\frac{\sqrt{c^{2}+\kappa}}{\left(a^{2}+\kappa\right)}\right]=0$,

and

$n^{2}-\frac{1-\mu}{r_{1}^{3}}-\frac{3 \mu}{2\left(a^{2}-c^{2}\right)} \times$

$\left[\frac{1}{\sqrt{a^{2}-c^{2}}} \sin ^{-1} \sqrt{\frac{a^{2}-c^{2}}{a^{2}+\kappa}}-\frac{\sqrt{c^{2}+\kappa}}{\left(a^{2}+\kappa\right)}\right]=0$,

On eliminating the last terms from the Eqns. (15) and (16), we have

$n^{2}-\frac{1}{r_{1}^{3}}=0 \Rightarrow r_{1}=n^{-2 / 3}=1-\frac{A}{2}$

Again, on eliminating $r_{1}$ from Eqns. (15) and (16), we now get

$n^{2}-\frac{3}{2\left(a^{2}-c^{2}\right)}\left[\frac{1}{\sqrt{a^{2}-c^{2}}} \times \sin ^{-1} \sqrt{\frac{a^{2}-c^{2}}{a^{2}+\kappa}}-\frac{\sqrt{c^{2}+\kappa}}{\left(a^{2}+\kappa\right)}\right]=0$

On simplifying Eqn. (18) and considering only linear terms in $a^{2}-$ $c^{2}$, we have

$n^{2}-\left(\frac{1}{\left(a^{2}+\kappa\right)^{3 / 2}}+\frac{3\left(a^{2}-c^{2}\right)}{10\left(a^{2}+\kappa\right)^{5 / 2}}+\ldots\right)=0$.

Since $A=\left(a^{2}-c^{2}\right) / 5<<1$, therefore considering only linear terms in $A$, we get

$n^{2}-\left(\frac{1}{\left(a^{2}+\kappa\right)^{3 / 2}}+\frac{3 A}{2\left(a^{2}+\kappa\right)^{5 / 2}}\right)=0$

Obviously, $a^{2}+\kappa=1$, is the solution of Eqn. (19). Thus, the noncollinear equilibria are the solution of the Eqns. $r_{1}=n^{-2 / 3}$ and $\kappa=1-a^{2}$ iff $r_{2}=1$ (Eqn. 6).

Hence, the coordinates of non-collinear equilibria $L_{4,5}$ are $x=\mu-\frac{1}{2}+\frac{A}{2}$, 


$$
y= \pm \frac{\sqrt{3}}{2}\left(1-\frac{A}{3}\right)
$$

Thus, there exist two non-collinear equilibria $L_{4,5}$ defined in Eqns. (20) and (21). The results are totally agree with [6] and [9]. Also, these points are linearly stable for critical mass parameter $\mu_{\mathrm{c}}=\mu_{\mathrm{o}}$ $-0.285001787791 A$, where $\mu_{\mathrm{o}}=0.038520896505$ [6].

\section{Conclusion}

In the present paper the existence of non-collinear equilibria in restricted three-body problem considering less massive primary an oblate spheroid has been discussed when potential is given in terms of $\kappa$, the largest root of confocal spheroid passes through at an external point $P(x, y, z)$ given by W. D. MacMillan, 1958. The mean-motion of the primaries obtained is in conformity with [6] and [9]. Also, at non-collinear equilibria, the results are in agreement with [6] and [9]

\section{References}

[1] MacMillan, W. D.: The theory of the potential. Dover Publications, New York (1958)

[2] Danby, J.M.A.: Stability of the triangular points in the elliptic restricted problem of three bodies. The Astronomical Journal, 69 (2) 165 - 172 (1964). http://dx.doi.org/10.1086/109254.

[3] Szebehely, V.: Theory of orbits, The Restricted Problem of three bodies. Academic Press, New York and London (1967).

[4] Vidyakin, V.V.: Stability of one particular solution for the motion of three homogeneous spheroids.Soviet Astronomy, 18, 116 (1974).

[5] Sharma, R.K.: Perturbations of Lagrangian points in the restricted three-body problem. Indian Journal of Pure and Applied Mathematics, 6, 1099-1102 (1975)

[6] Subbarao, P.V., Sharma, R.K.: A note on the Stability of the triangular points of equilibrium in the restricted three body problem. Astronomy and Astrophysics, 43, 381-383 (1975).

[7] Sharma, R.K., Subbarao P.V.: Stationary solutions and their charac teristic exponents in the restricted three-body problem when the more massive primary is an oblate spheroid. Celestial Mechanics and Dynamical Astronomy, 13, 137-149 (1976). http://dx.doi.org/10.1007/BF01232721.

[8] Choudhary R.K.: Libration points in the generalized elliptic restricted three body problem. Celestial Mechanics, 16, 411 - 419 (1977). http://dx.doi.org/10.1007/BF01229285.

[9] Bhatnagar, K. B., Hallan, P. P.: Effect of perturbed potentials on the stability of Libration points restricted problem. Celestial Mechanics and Dynamical Astronomy, 20(2), 95-103 (1979). http://dx.doi.org/10.1007/BF01230231

[10] Cid, R., Ferrer, S., Caballero, J.A.: Asymptotic solutions of the restricted problem near equilateral Lagrangian points. Celestial Mechanics and Dynamical Astronomy, 35, 189-200 (1985). http://dx.doi.org/10.1007/BF01227668.

[11] El-Shaboury, S.M.: Equilibrium solutions of the restricted problem of $2+2$ axisymmetric rigid bodies. Celestial Mechanics and Dynamical Astronomy, 50, 199-208 (1991) http://dx.doi.org/10.1007/BF00048764.

[12] Bhatnagar, K.B., Gupta, U., Bharadwaj, R.: Effect of perturbed potentials on the non-linear stability of Libration point L4 in the restricted problem. Celestial Mechanics and Dynamical Astronomy, 59, 45-374 (1994). http://dx.doi.org/10.1007/BF00692102.

[13] Selaru, D., Cucu-Dumitrescu, C.: Infinitesimal orbits around Lagrange points in the elliptic, restricted three-body problem. Celestial Mechanics and Dynamical Astronomy, 61 (4), 333 - 346 (1995) http://dx.doi.org/10.1007/BF00049514.

[14] Markellos, V.V., Papadakis, K.E., Perdios, E.A.: Non-linear stability zones around triangular equilibria in the plane circular restricted three-body problem with oblateness. Astrophysics and Space Science, 245, 157-164 (1996). http://dx.doi.org/10.1007/BF00637811.

[15] Subbarao, P.V., Sharma, R.K.: Effect of oblateness on the nonlinear stability of L4 in the restricted three-body problem. Celestial Mechanics and Dynamical Astronomy, 65, 291-312 (1997).

[16] Khanna, M., Bhatnagar, K.B.: Existence and stability of libration points in restricted three body problem when the smaller primary is a triaxial rigid body. Indian Journal of Pure and Applied Mathematics, 29 (10), 1011-1023 (1998).
[17] Khanna, M., Bhatnagar, K.B.: Existence and stability of Libration points in the restricted three body problem when the smaller primary is a triaxial rigid body and the bigger one an oblate spheroid. Indian Journal of Pure and Applied Mathematics, 30 (7), 721-733 (1999).

[18] Roberts, G.E.: Linear Stability of the Elliptic Lagrangian Triangle Solutions in the three-body problem. Journal of Differential Equa-

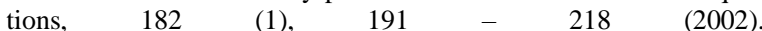
http://dx.doi.org/10.1006/jdeq.2001.4089.

[19] Oberti, P.,Vienne, A.: An upgraded theory for Helene, Telesto and Calypso. Astronomy and Astrophysics, 397, 353-359 (2003). http://dx.doi.org/10.1051/0004-6361:20021518.

[20] Sosnytskyi, S.P.: On the Lagrange stability of motion in the threebody problem. Ukrainian Mathematical Journal, 57, 1341 - 1349 (2005). http://dx.doi.org/10.1007/s11253-005-0266-8.

[21] Perdiou, A.E., Markellos, V.V., Douskos, C.N.: The Hill problem with oblate secondary: Numerical Exploration. Earth, Moon and Planets, 97, 127-145 (2005). http://dx.doi.org/10.1007/s11038-0069065-y.

[22] John, A. Arredondo, Jianguang, Guo, Cristina, Stoica, Claudia, and Tamayo: On the restricted three body problem with oblate primaries. Astrophysics and Space Science, 341, 315-322 (2012). http://dx.doi.org/10.1007/s10509-012-1085-7.

[23] Idrisi, M. Javed, Taqvi, Z.A.: Restricted three-body problem when one of the primaries is an ellipsoid. Astrophysics and Space Science, 348, 41-56 (2013). http://dx.doi.org/10.1007/s10509-013-1534-y.

[24] Idrisi, M. Javed: Existence and stability of the libration points in CR3BP when the smaller primary is an oblate spheroid. Astrophysics and Space Science, 354, 311-325 (2014). http://dx.doi.org/10.1007/s10509-014-2031-7.

[25] Idrisi, M. Javed, Amjad, Muhammad: Effect of elliptic angle $\varphi$ on the existence and stability of libration points in restricted threebody problem in earth-moon system considering earth as an ellipsoid. International Journal of Advanced Astronomy, 3 (2), 87-96 (2015). http://dx.doi.org/10.14419/ijaa.v3i2.5313. 